\title{
Determining Optimal Gear Ratios of a Two-stage Helical Reducer for Getting Minimal Acreage of Cross Section
}

\author{
Nguyen Khac Tuan ${ }^{1}$, Vu Ngoc $\mathrm{Pi}^{2}$, Nguyen Thi Hong $\mathrm{Cam}^{2}$, Tran Thi Phuong Thao ${ }^{2}$, Ho Ky Thanh ${ }^{2}$, Le Xuan Hung ${ }^{2}$, Hoang \\ Thi Tham ${ }^{3}$ \\ ${ }^{1}$ Automotive and Power Machinery Faculty, Thai Nguyen University of Technology, Thai Nguyen 23000, Vietnam
${ }^{2}$ Mechanical Engineering Faculty, Thai Nguyen University of Technology, Thai Nguyen 23000, Vietnam

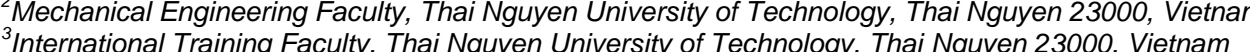

\begin{abstract}
This article introduces a study on the optimal calculation of gear ratios of a two-stage helical reducer. In the study, the acreage of the cross section of the reducer was chosen as the objective function of the optimization problem. Also, the design equation for pitting resistance of a gear set was investigated. Furthermore, the equations on moment equilibrium condition of a two-stage helical reducer and their regular resistance condition were analysed. A computer program was performed to determine the optimal partial transmission ratios for getting the minimal acreage of the cross section of the system. From the results of the computer program, two models for determining the optimal gear ratios of a two-stage reducer were proposed. Using these models, the gear ratios can be calculated accurately in a simple way.
\end{abstract}

\section{Introduction}

In optimum reducer design, the calculation of the gear ratios of the reducer plays a very important role. The reason is that the gear ratios strongly affect the size, the dimension, the mass, and the cost of the reducer. Subsequently, optimal determination of the gear ratios of reducers has been chosen by various researchers in their studies.

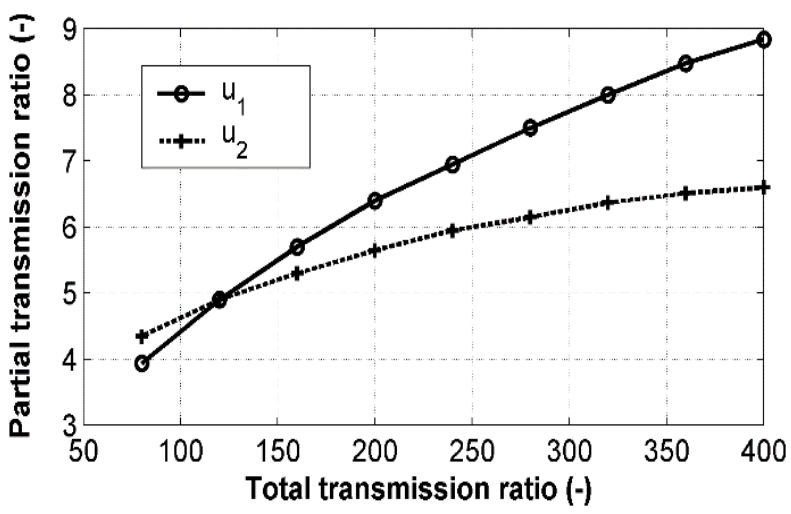

Figure 1. Gear ratios of step 1 and 2 versus the total gear ratio [1]

Up to now, there have been a number of optimization studies on determination of gear ratios of helical reducers. The gear ratios have been determined for two-stage reducers $[1,2,3$ and 4$]$, three-stage reducers $[4,5,6$, and $8]$, and four-stage reducers $[4,8,9$ and 10]. Also, the gear ratios have been found using different methods including designed experience method [11], graph method [1,2 and 4], "practical method" [2] and model method [5, 6, 7, 8, 9 and 10].

In designed experience method, the gear ratios can be found based on experiences in design and the gear ratios are not optimum. For example, for two-stage helical reducer, the relation between gear ratios of the first step $u_{1}$ and the second step $u_{2}$ follows the following equation [11]:

$$
u_{1}=(1.2 \mathrm{~K} 1.3) \cdot u_{2}
$$

For coaxial reducers, the gear ratios can be determined by the following equation [12]:

$$
u_{1}=u_{2} \approx \sqrt{u_{t}}
$$

Where, $u_{1}$ and $u_{2}$ are gear ratios of the first and the second stage; $u_{t}$ is the total ratio of the reducer.

In the graph method, the gear ratios were determined graphically. For example, for three-stage helical reducers, the gear ratios of the first stage and the second stage can be found from the graph in Figure 1 [1]. For coaxial reducers, the gear ratio of the first stage $u_{1}$ can be determined from the total ratio $u_{h}$ based on graph in Figure 2 [1]. The graph method was also used by Trinh 
Chat [13] for finding optimum gear ratios for two, three and four-stage helical reducers.

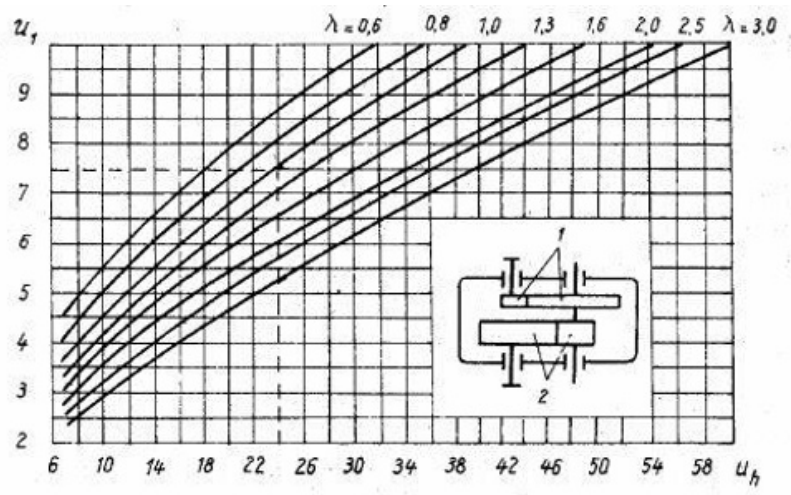

Figure 2. Gear ratio of step 1 versus the total gear ratio [1]

G. Milou et al. [2] proposed "practical method" for determining optimum gear ratios. In the "practical method", the gear ratios were found from practical data. For instance, based on the data from reducer factories, it was found that the weight of a two-stage reducer is minimal when the center distance ratio of the second stage to that of the first stage was from 1.4 to 1.6 [2]. After that, the optimal gear ratios were introduced in the tabulated form. For two-stage bevel helical gearboxes, the ratio between the bevel step and cylindrical step was 1.12 to 1.4 [2].

In the model method, the optimum gear ratios are calculated by models for different objectives. There have been quite a lot of studies which follow this method. In [5] and [14], the gear ratios were found in order to get the minimal cross section dimension of the gearbox. In [6], [9], [10] and [15], the objective for optimization problem was the minimum mass of gears. In [7], [16] and [17], the minimum gearbox length was chosen as the objective of the optimization problem for determining optimum gear ratios.

From above analysis, it is clear that till now there have been many studies on the determination of gear ratios of helical reducers. However, there is still lack of research on two-stage helical reducer which has the objective of minimal acreage of cross-section of the reducer. This paper presents a study on the optimal determination of the gear ratios for a two-stage helical reducer in which the objective is the minimal acreage of cross-section.

\section{Optimization problem for determining optimal partial transmission ratios}

The objective of the optimization problem is to determine the optimal gear ratios in order to get the minimal acreage of cross section of a two-stage helical reducer. From Figure 3, the acreage of cross section of the reducer $A$ can determination on two dimensions $L$ and $h$ and it can be calculated as:

$$
A=L \cdot h
$$

In which, $L$ and $h$ are determined as (see Figure 2):

$$
\begin{aligned}
& L=\frac{d_{\mathrm{w} 11}}{2}+a_{w 1}+a_{w 2}+\frac{d_{\mathrm{w} 22}}{2} \\
& h=\max \left(d_{w 21}, d_{w 22}\right)
\end{aligned}
$$
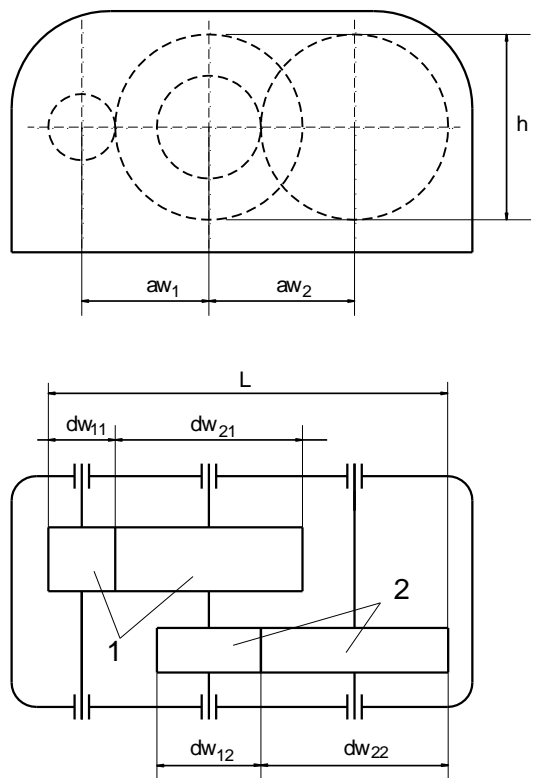

Figure 3. Calculation schema for optimum determination of gear ratio

In the above equations, $a_{w 1}$ and $a_{w 2}$ are the center distances of the first and the second stage $(\mathrm{mm}) ; d_{w 21}$ and $d_{w 22}$ are the gear pitch diameters of the first and the second stage $(\mathrm{mm}) ; d_{\mathrm{w} 11}$ is the pinion pitch diameter of the first step $(\mathrm{mm})$;

For a two-stage reducer, the diameters $d_{w 11}, d_{w 21}$ and $d_{w 22}$ can be determined as the following equations [18]:

$$
\begin{aligned}
& d_{w 11}=\frac{2 \cdot a_{w 1}}{u_{1}+1} \\
& d_{w 21}=\frac{2 \cdot a_{w 1} \cdot u_{1}}{u_{1}+1} \\
& d_{w 22}=\frac{2 \cdot a_{w 2} \cdot u_{2}}{u_{2}+1}
\end{aligned}
$$

Also, for a two-stage reducer we have:

$$
u_{2}=\frac{u_{t}}{u_{1}}
$$


In equations (6), (7), (8) and (9), $u_{1}$ is the gear ratio of the first stage; $u_{2}$ is the gear ratio of the second stage; $u_{t}$ is the total reducer ratio.

From (4), (5), (6), (7), (8) and (9), dimensions $L$ and $h$ can be expressed as functions of center distances and gear ratios of two stages. Therefore, the following equations are given:

$$
\begin{aligned}
& L=f\left(a_{w 1}, a_{w 2}, u_{1}, u_{t}\right) \\
& h=f\left(a_{w 1}, a_{w 2}, u_{1}, u_{t}\right)
\end{aligned}
$$

Thus, the optimization problem is defined as:

$$
\text { minimize } A=L \cdot h
$$

With the following constraints

$$
5 \leq u_{t} \leq 40
$$

From (10), (11), (12) and (13), it is clear that for solving the optimization problem it is necessary to calculate the center distances of the first stage $a_{w 1}$ and the second stage $a_{w 2}$.

\subsection{Determining the center distance of the first step}

From the contact strength condition, the center distance of the first step can be calculated by the following equation [18]:

$$
a_{\mathrm{w} 1}=K_{a} \cdot\left(u_{1}+1\right) \cdot \sqrt[3]{\frac{T_{11} \cdot k_{H \beta}}{\left[\sigma_{H}\right]^{2} \cdot u_{1} \cdot \psi_{b a 1}}}
$$

Where,

- $K_{H \beta}$ is the contact load ratio for pitting resistance; for the first step of a two-stage helical reducer $k_{H \beta}=1.02 \div 1.28$ [18]. Therefore, we can choose $k_{H \beta}=1.1$;

- $\left[\sigma_{H}\right]$ - the allowable contact stress $(\mathrm{MPa})$; In practice, $\left[\sigma_{H}\right]=350 \mathrm{~K} 410 \quad(\mathrm{MPa})$ and we can choose $\left[\sigma_{H}\right]=380(\mathrm{MPa})$;

- $k_{a}$ is the material coefficient; As the gear material is steel, $k_{a}=43[18]$;

- $\psi_{b a}$ is the coefficient of wheel face width; for the first step of a two-stage helical reducer $\psi_{b a}=0.3$;

- $T_{11}$ is the turning moment on the pinion of the first stage ( N.mm);

Substituting the above values into equation (14), the value of the center distance of the first stage can be found:

$$
a_{\mathrm{w} 1} \approx 1.2639 \cdot\left(u_{1}+1\right) \cdot \sqrt[3]{\frac{T_{11}}{u_{1}}}
$$

From the moment equilibrium condition of a two-stage helical reducer and their regular resistance condition, the output turning moment $T_{\text {out }}$ is can be calculated by the following equation:

$$
T_{\text {out }}=T_{11} \cdot \eta_{h g}^{2} \cdot \eta_{b e}^{3} \cdot u_{t}
$$

Wherein, $\eta_{h g}$ is the helical gear transmission efficiency ( $\eta_{h g}$ is from 0.96 to $\left.0.98[18]\right) ; \eta_{b e}$ is the transmission efficiency of a pair of rolling bearing ( $\eta_{b e}$ is from 0.99 to 0.995 [18]). Choosing $\eta_{h g}=0.97$ and $\eta_{b e}=0.992$ [18] gives

$$
T_{11}=1.0887 \cdot \frac{T_{\text {out }}}{u_{t}}
$$

\subsection{Determining the center distance of the second step $a_{w 2}$}

Calculating as in section 2.1 and with the note that the coefficient of wheel face width for the second stage $\psi_{b a}=0.35$, the center distance of the second stage is calculated by:

$$
a_{\mathrm{w} 2} \approx 1.2006 \cdot\left(u_{2}+1\right) \cdot \sqrt[3]{\frac{T_{12}}{u_{2}}}
$$

Also, for the second step we have

$$
T_{\text {out }}=T_{12} \cdot \eta_{h g} \cdot \eta_{b e}^{2} \cdot u_{2}
$$

Choosing $\eta_{h g}=0.97$ and $\eta_{b e}=0.992$ as in section 2.1 gives

$$
T_{12}=1.0476 \cdot \frac{T_{\text {out }}}{u_{2}}
$$

\section{Results and discussions}

Based on Equations (12) and (13), a computer program was performed to determine the optimal partial transmission ratios for getting the minimal acreage of the cross section of the system. The following data were used in the optimization program: $u_{t}=5 \mathrm{~K} 40$; $T_{\text {out }}=10^{6} \mathrm{~K} 10^{7}$.

From the results of the optimization program, the relation between the gear ratios of two stages $\left(u_{1}\right.$ and $\left.u_{2}\right)$ with the total reducer ratio $u_{t}$ is described as in Figure 4. From the figure, it is clear that with the increase of the total 
ratio the gear ratio of the first stage grows faster than that of the second stage. This is because the torque on the pinion of the first stage is lower than that of the second stage. It was also found that the output torque $T_{\text {out }}$ does not affect the gear ratios.

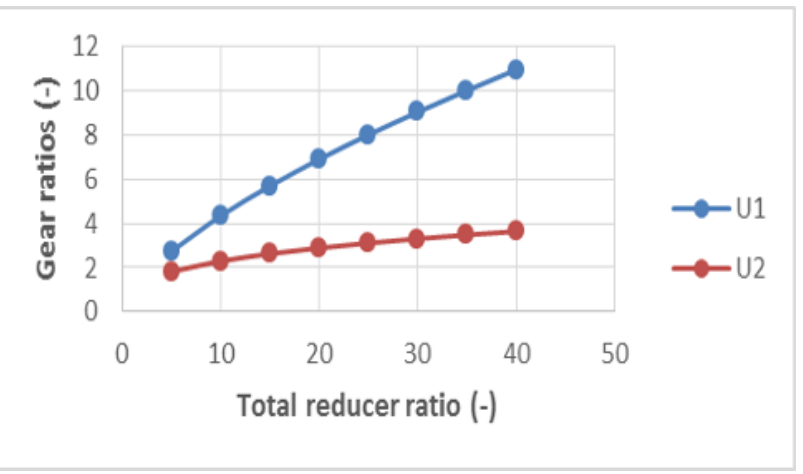

Figure 4. Gear ratios versus total reducer ratio

From the results of the program, the following regression model were proposed in order to determine the gear ratio of the first stage:

$$
u_{1}=0.9365 \cdot u_{t}^{0.667}
$$

The above equation fit very well with the data. The coefficient of determination for the equation was $R^{2}=1$. Equation (20) is used to calculate the gear ratio of the first stage of the reducer. After determining $u_{1}$, the gear ratio of the second stage is determined by:

$$
u_{2}=\frac{u_{t}}{u_{1}}
$$

\section{Conclusions}

The minimal cross-sectional acreage of a two-stage helical reducer can be found by optimal determination of gear ratios of two stages of the reducer.

The models for calculation of the gear ratios of the helical reducer for getting the minimal cross-sectional acreage of the reducer were proposed.

The gear ratios of the reducer can be calculated accurately and simply by using explicit equations.

\section{Acknowledgements}

The work described in this paper was supported by Thai Nguyen University of Technology for the project N0 T2018-B17.

\section{References}

1. V.N. Kudreavtev; I.A. Gierzaves; E.G. Glukharev, Design and calculus of gearboxes (in Russian), Mashinostroenie Publishing, Sankt Petersburg, 1971.

2. G. Milou; G. Dobre; F. Visa; H. Vitila, Optimal Design of Two Step Gear Units, regarding the Main Parameters, VDI Berichte No 1230 (1996), p. 227.
3. Vu Ngoc Pi, A method for optimal calculation of total transmission ratio of two step helical gearboxes, Proceedings of the National conference on Engineering Mechanics, Ha Noi (2001), p. 12.

4. A.N. Petrovski, B.A. Sapiro, N.K. Saphonova, About optimal problem for multi-step gearboxes (in Russian),Vestnik Mashinostroenie, No. 10 (1987), p. 13.

5. Vu Ngoc Pi, Nguyen Khac Tuan, Optimum determination of partial transmission ratios of Threestep helical gearboxes for getting minimum cross section, Journal of Environmental Science and Engineering A 5 (2016) 570-573.

6. Vu Ngoc Pi, Nguyen Dang Binh, Vu Quy Dac, Phan Quang The, "Optimal Calculation of Total Transmission Ratio of Three-Step Helical Gearboxes for Minimum Mass of Gears." (In Vietnamese) Journal of Science and Technology of 6 Engineering Universities (2006), p. 91.

7. $\mathrm{Vu}$ Ngoc Pi, A new study on optimal calculation of partial transmission ratio of three-step helical reducers, The 3rd IASME / WSEAS International Conference on Continuum Mechanics, Cambridge, UK (2008), pp. 10-13.

8. Romhild I. , Linke H., Gezielte Auslegung Von Zahnradgetrieben mit minimaler Masse auf der Basis neuer Berechnungsverfahren, Konstruktion 44, 1992, pp. 229- 236.

9. $\mathrm{Vu}$ Ngoc Pi, Optimal determination of partial transmission ratios for four-step helical gearboxes with firt and third step double gear-sets for minimal mass of gears, Applied Computing Conference (ACC' 08), Istanbul, Turkey, May 27-30, 2008.

10. Le Xuan Hung, Vu Ngoc Pi, Nguyen Van Du, Optimal calculation of partial transmission ratios of four-step helical gearboxes with second and fourthstep double gear-sets for minimal mass of gears, The international symposium on Mechanical Engineering (ISME Ho Chi Minh city, Vietnam, 21-23, September 2009.

11. Nguyen Trong Hiep, Nguyen Van Lam, Design of machine elements (in Vietnamese), Educational Republishing House, Hanoi, 1979.

12. C.A. Trernapxki, G.A. Trekharev, Design of Mechanical Transmissions (in Russian), Mashinostroenie Publish, Moskova, 1984.

13. Trinh Chat, Optimal calculation the total transmission ratio of helical gear units (in Vietnamese), Scientific Conference of Hanoi University of Technology (1996) 74-79.

14. Vu Ngoc Pi, A new study on optimal calculation of partial transmission ratios of three-step helical reducers for getting minimal cross section dimension, The $2^{\text {nd }}$ WSEAS International Conference on Computer Engineering and Applications (CEA'08), Acapulco, Mexico January 25-27, 2008, pp. 290-293.

15. Vu Ngoc Pi, Optimal determination of partial transmission ratios of three-step helical gearboxes with first and third-step double gear-sets for minimal mass of gear, American Conference on Applied Mathematics (MATH'08), Harvard, Massachusetts, USA March 24-26, 2008, pp. 385-388. 
16. Vu Ngoc Pi, Optimal calculation of partial transmission ratios for four-step helical gearboxes with first and third-step double gear-sets for minimal gearbox length, American Conference on Applied Mathematics (MATH'08), Harvard, Massachusetts, USA March 24-26, 2008, pp. 29-32.

17. Vu Ngoc Pi, Optimal calculation of partial ratios of helical gearboxes with second-step double gear-sets,
The 3rd IASME / WSEAS International Conference on Continuum Mechanics, Cambridge, UK (2008), pp. 63-66.

18. Trinh Chat, Le Van Uyen, Design and calculus of Mechanical Transmissions (in Vietnamese), Educational Republishing House, Hanoi, 1998. 